\title{
A Study on Translation from the Perspective of Memetics
}

\author{
Huang Huannai $1, a^{*}$ \\ Xi'an Technological University \\ Xi'an, Shaanxi, China \\ Email:915677282@qq.com
}

\begin{abstract}
Studying from the perspective of memetics and combining with Chesterman's theory of translation strategies, the employment of translation strategies and techniques is regarded as a way of copying original meme (complexes) form three levels, including syntax, semantics and pragmatics. Then this paper proves the rationality of applying Heylighen Francis's general selection criteria for strong memes, such as formality, coherence, self-justification, into the evaluation of translation effect, aiming to make a closer connection between memetics and translation practice from two aspects of input and output, and providing a humble enlightenment for probing the optimization of translation strategies and techniques.
\end{abstract}

Keywords-Memetics; Translation; Strategies and techniques; evaluation criteria

\section{INTRODUCTION}

In 1976, Richard Dawkins, a zoologist in University of Oxford, was the first person to put up with "meme" by imitating "gene" in Selfish Gene, and pointed out that meme is a unit of culture transmission, or the replicator of culture, as well as an "imitation unit".

In 1997, Andrew Chesterman, a Finland scholar, and Hans Vermeer, a famous German translation theorist, first introduced the concept of meme into translation research. But it was the book Memes of Translation written by Andrew Chetsman that truly had a great effect on translation teaching and practice.

In 1998, Francis Heylighen, a research professor at Vrije Universiteit Brussel (VUB), designed a four-stage model to analyze the replication mechanism and life cycle of memes in the article what makes a meme successful? Selection criteria for cultural evolution.

In 2005, Kate Dustin proposed in The Selfish Meme that memetics provided a new explanation for the origin of language and this was a very important element in human culture. Kate Dustin deepened and broadened the theory of Dawkin in this book, coming up with a new and practical concept of cultural DNA in which both memetic evolution and products of human creativity promoted the development of culture.

\author{
Zhao Zhuoxing 2,b \\ Zhengzhou Institute of Finance and Economics, Zhengzhou, \\ Henan, China \\ Email: 907901383@qq.com
}

So far there have been many meme centers, meme laboratories, topic websites, online discussion groups and meme journals at abroad. Meme has been widely spread into the fields of psychology, sociology, culture, philosophy, language teaching, etc..

\section{RELATED TERMS ABOUT MEME}

Meme, as the nuclear of memetics, is originated from a Greek word "mimeme" which means imitation and refers to the unit of cultural transmission or the unit of replication. Like genes, memes have their own living environment and the law of survival and spread, survival of the fittest and strongest. From the perspective of successful transmission, meme can be divided into two categories: strong memes and weak memes. At the same time, the transmission of memes also needs carriers, such as language, and hosts, such as the reader or translator (He Ziran, 2005)

Meme Pool refers to the collection or aggregation of various memes contained in the same environment, such as society, culture or language, context.

Meme complexes is the abbreviation of "common adaptation meme complex" called by Dawkins, which means that a group of memes that can work together and complement each other like genes. In translation, the source language meme complex mainly consists of memes of author's ideas, memes of source language and memes of source language culture; target language memes complex mainly consists of memes of integrate ideas of the translator and the original author, memes of source culture expressed in the target language, memes of target language and memes of target language culture (Pan Xiaobo, 2011). To some extent, a word is a language meme complex, consisting of syntactic meme, syntactic meme and pragmatic meme.

\section{ANDREW CHESTERMAN'S TRANSLATION STRATEGIES}

Based on the metaphor of "meme", Chesterman divides translation strategies and techniques into the following three levels and more than thirty sub-types. (See Table 1) 
TABLE I CHESTERMAN'S TRANSLATION STRATEGY

\begin{tabular}{|l|l|l|l|}
\hline Level & Syntactic strategies & Semantic strategies & Pragmatic strategies \\
\hline Sub-types & G1Literal translation & S1Synonomy & Pr1Cultural filtering \\
& G2Loan, calque & S2Antonomy & Pr2Explicitness change \\
& G3Transposition & S3Hyponomy & Pr3Information change \\
& G4Unit shift & S4Converses & Pr4Interpersional \\
& G5Phrase structure change & S5Abstraction change & Pr5Illocutionary change \\
& G6Clause structure change & S6Distribution change & Pr6Coherence change \\
& G7Sentence structure change & S7Emphasis change & Pr7Partial change Pr8Visibility \\
& G8Cohesion change & S8Paraphrase & change \\
& G9Level shift & S9Trope change & Pr9Transediting \\
& G10Scheme change & S10Other semantic changes & Pr10Other pragmatic changes \\
\hline
\end{tabular}

Syntactic/grammatical strategies refer to the complete or partial duplicationlvariation of the form of the source language only through syntactic or grammatical changes. For example, literal translation is similar to the meaning of "maximally close to the SL form, but nevertheless grammatical"; transposition, borrowed form Vinay and Darbelnet, is used to change word class from adjective to noun, from noun to verb, etc.; unit shift is a translation technique by which the source language unit is translated into a different unit in the target language. Here the units include morpheme, word, phrase, clause, sentence, paragraph, that is to say, a phrase in the SL may be translated into a clause in the TL.

The semantic strategy means a complete or partial duplicationlvariation of the meaning of the source language by means of semantic changes. For instance, hyponymy refers to shifts within the hyponymy relation: superordinate and its hyponyms, that is to say, a superordinate in SL may be translated into one of its hypomyms in TL, and vice versa; converses mean pairs of verbal structures expressed for the same thing from an opposing perspective. And one part of the pairs in SL may be translated into the opposite part in TL; cohesion change refers to the change in intra-textual reference, substitution, repetition, ellipsis etc..

A pragmatic strategy is used to make a bigger change of the source language, which contains a combination of grammatical and semantic changes and this strategy not only involves translator's macro decision on how to translate better, but also takes into account translator's view of the reader: catering to reader's expectations or sticking to the faithfulness in translation. Examples are given as follows: cultural filtering is a means through which SL items, particularly, culturally loaded terms, are translated as equivalents in TL culture according to TL norms; information change indicates the addition of new information, relevant to TL readers for better understanding or the omission of SL information, irrelevant or non-existent in TL culture for higher readability; explicitness, one of the most common translation strategies, is the way in which implicit information is translated overtly by adding explicit components. At the same time, implication works the opposite way by leaving some elements of the massage implicit.

The source of Chesterman's strategy classification and examples mostly comes from Vinay and Darbelnet's concept of modulation, Catford's theory of translation shift, Nida's translation theory, Joseph Malone's "transmission" mode, etc.. "I have drawn on all these in setting up my own classification". "Some of the classifications proposed have been extremely simple, such as Nida's with four classes only: change of word, omission, change of structure, addition. Other's are more complex, and hence less 'portable': Malone's approach, which uses a highly idiosyncratic terminology, would seem difficult to use in practice without a long apprenticeship." (Chesterman, 2012) Chesterman's classification has become more simple and easier to understand, making this theory represented and operationalized.

\section{MEME TRANSMISSION AND LANGUAGE TRANSLATION}

There is a close relationship between meme transmission and language translation.

Meme transmission mechanism and translation process

In 1998, Francis Heylighen designed a four-stage model to analyze the replication mechanism and life cycle of memes, including assimilation, retention, expression and transmission [3]. Similarly, the translation of source language memes also needs to experience four stages, which can be demonstrated as follows: source language is abbreviated as SL; target language as TL. (See Fig 1) 


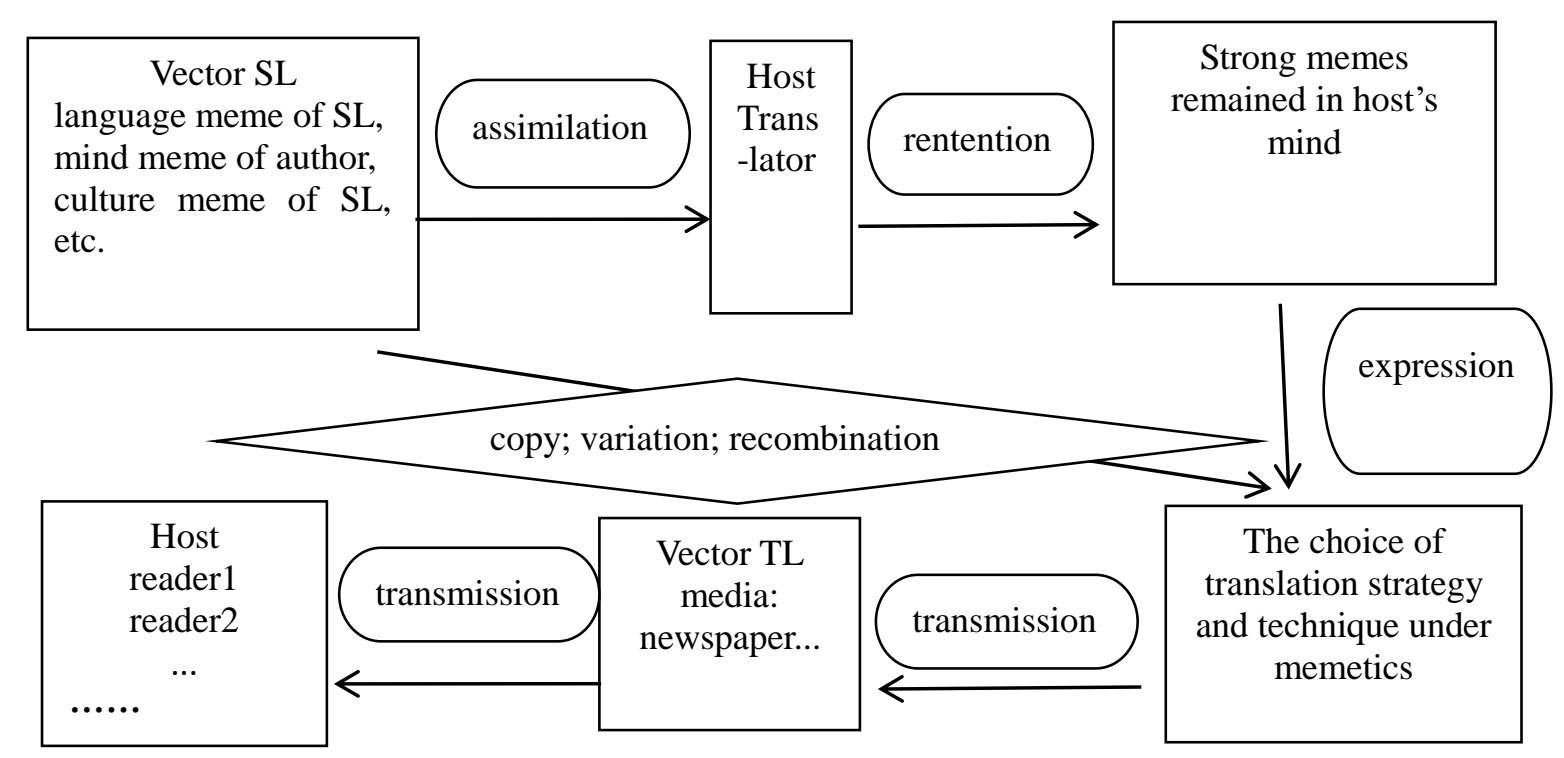

Fig. 1 Four-stage Translation process

General selection criteria for memes and translation effect

Taking account of four stages memes go through during spread, Heylighen put up with a series of general selection criteria for memes form the angles of meme itself, object and subject (see Table 2).

TABLE II GENERAL SELECTION CRITERIA FOR MEMES

\begin{tabular}{|l|l|l|l|l|}
\hline Stage & Objective & Subjective & Inter-subjective & Meme-centered \\
\hline assimilation & distinctiveness & novelty & authority & self-justification \\
rentention & invariance & simplicity & formality & self-reinforcement \\
expression & controllability & coherence & conformity & intolerance \\
transmission & & coherence & expressivity & proselytism \\
& & utility & publicity & proselytism \\
\hline
\end{tabular}

According to Heylighen, the more a meme conforms to these selection criteria, the greater the possibility of successful replication is. Similarly, only when the translated work is the carrier of the strong language memes according to these selection criteria, can it be transmitted successfully in the meme pool of target language, which is the best translation effect most translators intend to achieve. Therefore, general selection criteria is justified for the assess of translation effect.

But in terms of maneuverability, it is unrealistic and unnecessary to apply all of the 16 standards, put forward by Heylighen under ideal conditions, into the prediction of translation effect. In addition, there exits repetition and compatibility among these standards. Therefore, the author suggests that in actual application, researchers can choose 3 to 5 standards based on their main tasks and research, taking into account three angles of meme itself, the object, along with four stages of meme transmission.
V. CONCLUSION

In this paper, the theory of Chesterman's translation strategies is introduced to make close connection between selection of translation strategies, techniques and copy patterns of meme transmission. In the process of translating, the translator tries to adapt the form, meaning and pragmatic function of source language memes to the meme pool of target language memes in order to achieve largest transmission. It also is proved in this paper that Heylighen's general selection criteria for memes can be used to predicate the translation effect: only the translated version contain more strong memes, can it be successfully replicated and spread in the meme pool of target language through observing selection criteria. To sum up, this paper makes a closer relationship between memetics and translation practice from two aspects of input and output. 


\section{ACKNOWLEDGMENT}

This paper is supported by the fund of Xi'an Technological University (XAGDYJ170225).

\section{REFERENCES}

[1] Dawkins Richard, The Selfish Gene [M]. Oxford: Oxford University Press, 1976.

[2] Chesterman Andrew, Memes of Translation: The Spread of Ideas in Translation Theory [M]. Shanghai: Shanghai Foreign Language Education Press, 2012.

[3] Heylighen Francis, What Makes a Meme Successful? Selection Criteria for Cultural Evolution [J]. In Ramaekers, Jean, Eds.Proceedings 15th International Congress on Cybernetics, 1998.

[4] Distin Kate, The Selfish Meme [M]. Cambridge: Cambridge University Press, 2005.

[5] Pan Xiaobo, A Study on the Development, Existing Problem and Suggestions for Further Research of Memetics [J]. Journal of Zhejiang International Studies University, 2011. (In Chinese)

[6] He Ziran, Memes in language [J]. Linguistic Science, 2005. (In Chinese)

[7] Han Hongjiang, The introduction of Chesterman's translation norms [J]. Foreign Language Research, 2004. (In Chinese) 